\title{
How to make a good impression (crown and bridge)
}

\author{
Brian Millar BDS, FDSRCS, PhD, Senior Lecturer, Consultant in Restorative Dentistry, Department \\ of Conservative Dentistry, GKT Dental Institute, King's College London
}

Q. What are the steps in achieving a successful impression?

A. There are several key factors in obtaining a good impression:

- Prepare the soft tissues

- Select an impression technique

- Select an impression material

- The impression recording procedure

Q. How can I prepare the soft tissues?

A. Aim for:

- Good soft tissue health beforehand

- Ensure that the preparation margin is visible, perhaps best achieved by recontouring the gingival margin using electrosurgery.

- Placing margins supragingivally is an advantage but where this is not desirable then retraction cord can be used.

Q. Which impression technique should I consider?

A. The choice is usually between putty/wash, dual-phase (heavy and light) or monophase.

The majority of UK dentists use a one-stage putty-wash technique with the advantages of simplicity, reasonable economy, predictability and dimensional accuracy ${ }^{\top}$ as well as speed, particularly when a fast set material is used. Selection of a metal or rigid tray allows for greater accuracy. ${ }^{2}$

What typically happens (see Fig. la,b) is the putty tends to push the light body wash off the prepared tooth. The wash material then ends up in the lingual or buccal sulcus and the

Fig. la,b Typical one-stage putty wash impressions illustrating how the putty pushes the light body off the preparations, including key areas such as the margins of preparations
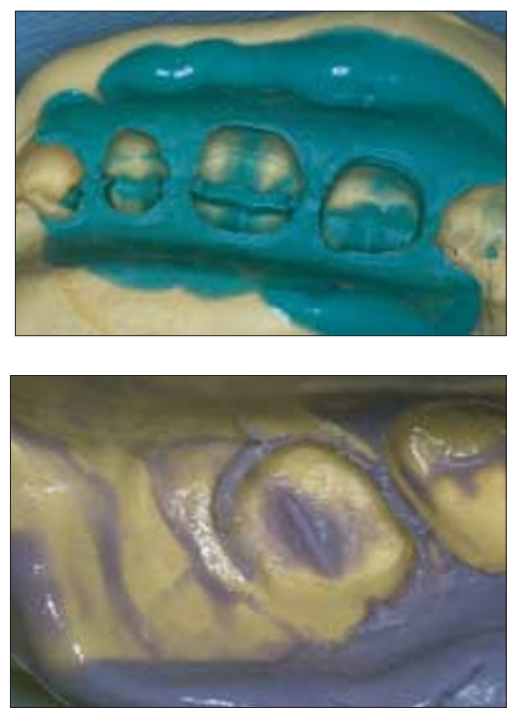

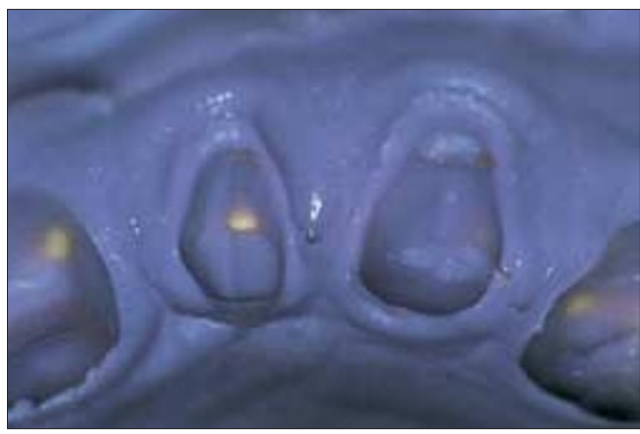

Fig. 2 A two-stage putty wash impression with the same materials as in Fig. 16

critical areas, such as preparation margins, may be recorded with putty which cannot record detail as well as the wash material. This is a particular problem where the preparation margin is towards the occlusal surface of the tooth, eg inlays, onlays, 3/4 crowns, porcelain veneers, resin bonded bridges.

Another difficulty with the technique is that once the light body is on the preparation the putty needs to be brought into position and seated. During this critical phase the patient may bring their tongue or floor of the mouth close to the teeth and remove the light-body material from the tooth. This can be a problem with the lingual aspect of lower molar teeth.

Q. So what are the alternative techniques to overcome these problems?

A. One method is to record the putty-wash impression in two steps of which there are several options:

Putty then wash as two separate stages

It is useful to record the putty before preparing the tooth. This will give some space for the wash material around the preparation otherwise there may be distortion of the putty or tray as the wash material is compressed. Figure 2 shows an example of a two-stage putty wash.

The disadvantages of this two-stage technique include the additional time of having to wait for two materials to set, contamination of the putty with saliva which may prevent light body adhering to it, and difficulty in reseating the set putty in the mouth.

Sluices are usually cut into the putty running away from the prepared tooth regions. A scalpel can be used to cut V-shaped sluices but a putty knife makes the job quick and easy (Fig. 3). The sluices allow for excess material to escape. If necessary

\section{SPONSORED BY AN EDUCATIONAL GRANT FROM}




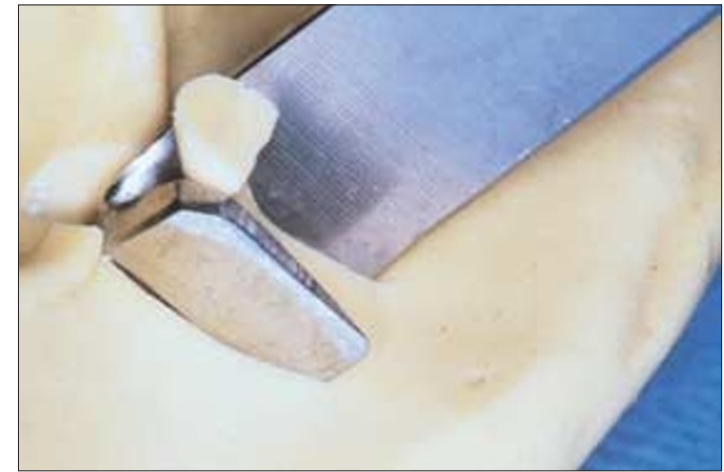

Fig. 3 Cutting a sluice channel with a putty knife.

the area around the prepared tooth can also be cut away, for example, if the putty impression was recorded after the tooth was prepared.

The putty can also be used to make the temporary restoration before reusing the putty for the final impression provided any residue from the bis-acryl type of temporary materials is removed.

One problem with this method is the creation of an occlusal step as some light-body spreads along the occlusal surface during reseating of the putty. As the tray begins to seat the light body material is compressed, becoming less viscous and escapes along the occlusal surfaces until the tray has seated. So this modification to the putty-wash technique has not solved all the problems!

The ideal might seem to be seating the putty first, thereby displacing the tongue, and then place the wash material which cannot then pass along the occlusal plane.

Q. How can this be achieved?

A. By the 'injection moulded' method.

This technique is the author's modification of a procedure described for use with dual arch impression trays ${ }^{3}$ which are not generally accepted in the UK by dentists or laboratories:

- A preoperative full-arch putty impression is recorded (usually while the local anaesthetic is taking effect) in a rigid plastic tray

- Prepare the putty (trim if necessary to ease reinsertion) and use to make the temporary restoration. The temporary restora-

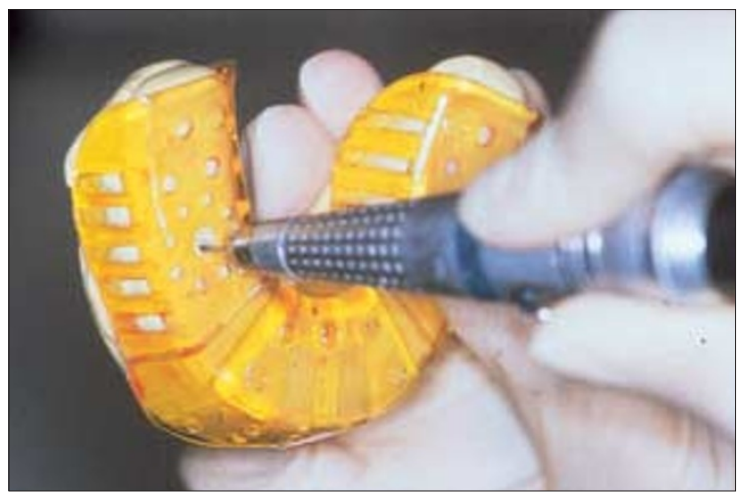

Fig. 4 Cutting the injection hole through the putty impression for the injection moulding technique tion can be used as a guide to your final restoration and to confirm occlusal clearance and amount of tooth reduction

- The putty is then modified by drilling a hole through the putty to the outside of the tray through one of the tray perforations (Fig. 4). It is also useful to cut away putty in the region of the gingival margin to allow for greater flow of light-body as well as a buccal 'escape' channel. Figure 5 illustrates the modified putty impression ready for injection moulding.

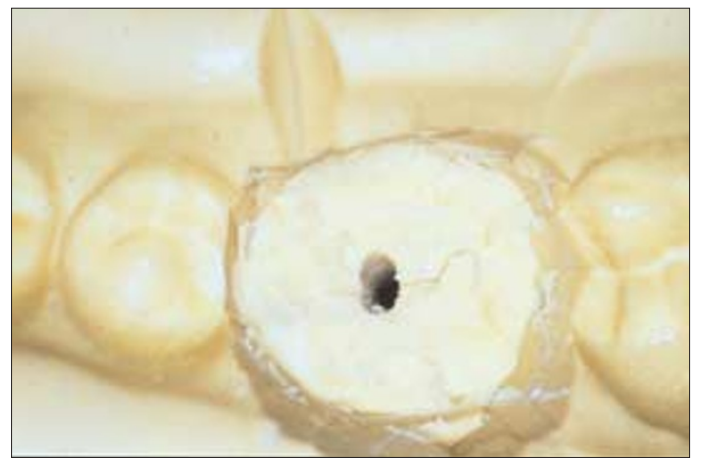

Fig. 5 Putty modified for the injection moulding technique by cutting away putty from around the prepared tooth.

- The tooth is cleaned (remove Vaseline if used for the temporary restoration), retraction cord removed, and the tooth is washed and dried.

- The putty is reinserted and checked for position on the teeth.

- A very small volume of light-body is mixed and placed in a syringe, or an auto-mix cartridge is used, and injected through the hole onto the preparation. It can soon be seen escaping buccally along the escape channel. The tray must be held down while injecting to prevent it lifting off the teeth (Fig. 6).

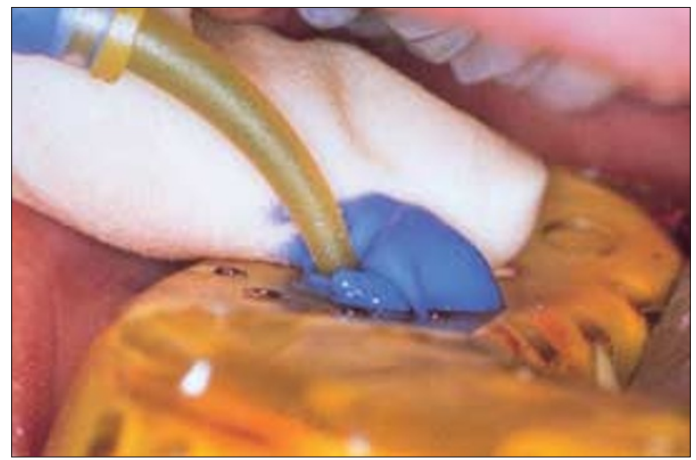

Fig. 6 Injecting the light body through the reinserted putty impression while holding the tray in position. Only a very small volume of light body is required.

The main advantages of the technique are:

1. It can be used with single arch or dual arch trays (but not metal trays!)

2. Ideal where soft tissues, usually the tongue, impedes access to the tooth.

3. Economical

4. Suited to the recording of multiple prepared teeth

5. Can be used to repair impressions

\section{DENSPLY




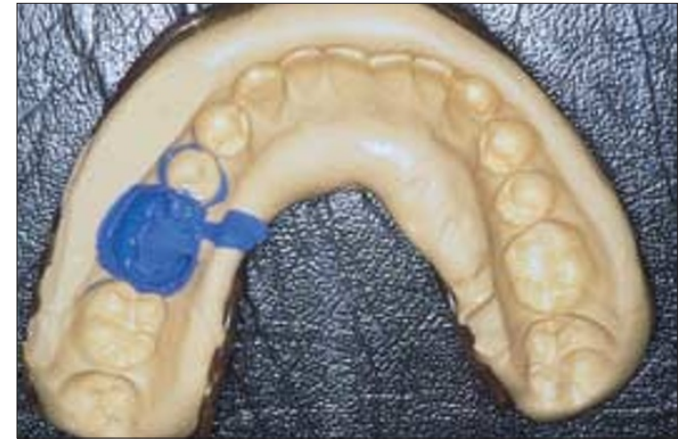

Fig. 7 An injection moulded impression.

Q. Are there any other alternative techniques?

A. Other techniques in use are:

Putty with a spacer sheet, then wash

The putty is first inserted into the mouth with a spacer sheet on the putty surface. This spacer prevents the putty taking a detailed impression of the teeth. It also prevents contamination of the putty by saliva while conveniently providing sluices in the putty. The putty is removed immediately and allowed to set outside the mouth (Fig. 8). Once polymerised the polythene sheet is peeled away prior to recording the final impression when a light body material is placed on the teeth and in the putty trough. More light body will be needed for this method but all the teeth will be recorded in light body with high detail.

\section{Dual-phase}

The most accurate impression is usually achieved using heavy body and light body addition silicone in conjunction with a rigid custom tray and a meticulous clinical technique. However, these materials can also be used in a rigid stock tray.

\section{Monophase}

Similar to dual-phase but with the same material for the tray as syringed over the preparation. However, there is a greater incidence of voids in monophase impressions when compared with the two-phase technique. ${ }^{4}$

Q. How to select a suitable material?

A. This depends partly on the selected impression technique. The addition cured silicone materials offer:

1. The greatest accuracy and dimensional stability of impression materials

2. The greatest resistance to dimensional change following disinfection/sterilisation

3. No unpleasant taste or smell

4. A wide range of viscosities to suit different techniques

5. Similar wettability to polyether (Impregum $)^{5}$

6. Automixing which reduces the incidence of voids. ${ }^{6} \mathrm{~A}$ small diameter syringe tip is also beneficial. ${ }^{7}$

7. No restrictions on pouring within a specific time period The dentist may select a particular manufacturer's product where particular features are required. Tear resistance, a vis-

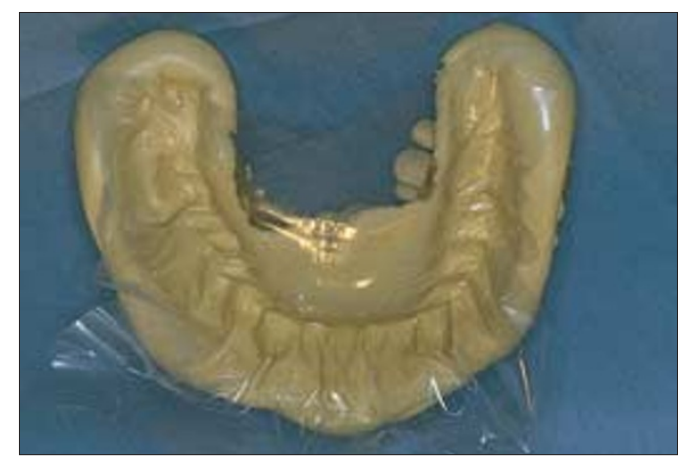

Fig. 8 Putty impression recorded with a polythene spacer sheet, now setting prior to reinsertion with light-body.

cosity to suit your impression technique and personal preference are likely to be key factors when selecting a material.

Hydrophilic materials are popular but should not be seen as an alternative to good moisture control and haemostasis.

When higher levels of surfactant are added to impression materials to make them more hydrophilic there is a significant reduction in tear strength ${ }^{8}$ and a slight loss of dimensional stability. ${ }^{9}$ However, the addition silicones exhibit the greatest resistance to tearing ${ }^{10}$ and dimensional stability following disinfection. ${ }^{11}$

\section{Conclusion}

A good quality impression is a prerequisite to the fabrication of well-fitting laboratory made restorations. The oral cavity offers many challenges to the clinician when it comes to recording accurate detail in a moist environment. Selection of a reliable technique and appropriate materials will assist in the recording of impressions. This article summarises popular impression techniques.

\section{References}

1. Hung $\mathrm{S} \mathrm{H}$ et al. Accuracy of one-step versus two-step putty wash addition silicone impression technique. J Prosthet Dent 1992; 67 583-589.

2. Carrotte $P$ V , Johnson A, Winstanley R B. The influence of the impression tray on the accuracy of impressions for crown and bridge work - an investigation and review. Br Dent J 1998; 185: 580-585.

3. Schoenrock G A. The laminar impression technique. J Prosthet Dent 1989; 62: 392-395.

4. Millar B J, Dunne S M, Robinson P B. In vitro study of the number of surface defects in monophase and two-phase addition silicone impressions. J Prosthet Dent 1998; 80: 32-35.

5. Pratten $\mathrm{OH}$, Craig R G. Wettability of a hydrophilic addition silicone impression material. J Prosthet Dent 1989; 61:197-202.

6. Soh G, Chong Y H. Defects in automixed addition silicone elastomers prepared by putty-wash impression technique. J Oral Rehabil 1991; 18: $547-553$.

7. Stackhouse J A. Relationship of syringe-tip diameter to voids in elastomer impressions. J Prosthet Dent 1985; 53: 812-815.

8. Millar B J, Wood D J, Bubb N L, Gabrielson L. Tear strength of hydrophilic and hydrophobic polyvinylsiloxane materials. J Dent Res $1995 ; 74: 881$, abstract 472 .

9. Panichuttra R et al. Hydrophilic poly(vinyl siloxane) impression materials: dimensional accuracy, wettability, and effect on gypsum hardness. Int J Prosthodont 1991; 4: 240-248.

10. Hondrum S O. Tear and energy properties of three impression materials. Int $J$ Prosthodont 1994; 7: 517-521.

11. Oda Y, Matsumoto T, Sumii T. Evaluation of dimensional stability ofelastomeric impression materials during disinfection. Bull Tokyo Dent Coll 1995; 36: 1-7.

\section{DENSPLY}

\section{UNITED}

